\title{
Intermédialités
}

Histoire et théorie des arts, des lettres et des techniques

Intermediality

History and Theory of the Arts, Literature and Technologies

\section{Night-clubs et discothèques : visions d'architecture}

\section{Carlotta Darò}

Numéro 14, automne 2009

bâtir

build

URI : https://id.erudit.org/iderudit/044411ar

DOI : https://doi.org/10.7202/044411ar

Aller au sommaire du numéro

Éditeur(s)

Revue Intermédialités (Presses de l’Université de Montréal)

ISSN

1705-8546 (imprimé)

1920-3136 (numérique)

Découvrir la revue

Citer cet article

Darò, C. (2009). Night-clubs et discothèques : visions d'architecture.

Intermédialités / Intermediality, (14), 85-103. https://doi.org/10.7202/044411ar
Résumé de l'article

Night-clubs et discothèques représentent, au cours des années 1960 et 1970, bien plus qu'un programme architectural à la mode. Cet article explore la manière dont, pour la néo-avant-garde de cette période, ces lieux de divertissement musical deviennent des laboratoires d'expérimentation stylistique, fonctionnelle, ainsi que des modèles et des inspirations d'ordre social. Un choix de projets représentatifs de cette époque - des pipers des architectes radicaux italiens aux visions urbaines de Constant et d'Archigram - montre une progression dans l'échelle adoptée, mais aussi d'ordre idéologique. Fascinée par l'exploitation de la technologie moderne et l'univers émergeant de la culture pop, cette génération d'architectes et d'artistes déplace son intérêt pour la forme bâtie vers un idéal du contrôle des environnements. 


\title{
Night-clubs et discothèques: visions d'architecture
}

\author{
Carlotta Darò
}

Nightclubbing we're nightclubbing

We're walking through town

Nightclubbing we're nightclubbing

We walk like a ghost

We learn dances brand new dances

Like the nuclear bomb

When we're nightclubbing

Bright white clubbing

Oh isn't it wild

Iggy Pop $(1977)^{1}$

D'après l'Oxford English Dictionary, une première trace d'utilisation du terme night-club apparaît en 1871, dans l'Appletons' Journal, pour décrire un club londonien nocturne avec salle de billard. Au fil du temps, le terme désigne tout local de nuit offrant en particulier un environnement musical. En anglais, le substantif a assumé aussi une valeur de verbe - to nightclub - indiquant la forme de divertissement associé à ce lieu. Night-club a été par la suite emprunté par d'autres langues dont le français. L'étymologie du terme discothèque, quant à elle, vient de l'assemblage de disco et thèque. Ce mot entra dans l'usage, d'après Le Petit Robert de la langue française, en 1928, pour désigner une collection de disques. Ce n'est que vers les années 1960, toujours d'après la référence française, que cela désigne aussi un lieu de réunion où l'on peut danser au son d'une musique enregistrée ${ }^{2}$. Le terme a été, pour sa part, adopté par la langue

1. Extrait de la chanson Nightclubbing sur l'album The Idiot d'Iggy Pop.

2. Par ailleurs, selon Jim Dawson, un night-club appelé La discothèque ouvrit ses portes, dès la fin des années 1930, dans la capitale française. Voir Jim Dawson, The Twist: 
anglaise. Ainsi, on retrouve plus précisément trace de son usage, d'après l'Oxford English Dictionary, dans un numéro du New Yorker de 1954 mentionnant des night-clubs de Saint-Germain-des-Prés, à Paris, où une musique pour danser était jouée par des phonographes. Le juke-box, machine qui fait automatiquement jouer des disques, était aussi utilisé dans ces premiers lieux de diffusion musicale enregistrée. Par ailleurs, l'utilisation du terme discothèque pour désigner la boîte de nuit coïncide avec un moment particulier d'éclosion de plusieurs de ces lieux. $\mathrm{La}$ « disco» devient un lieu de rencontres et une pratique de divertissement très à la mode, notamment parmi les noctambules des sixties.

Dans cet article, je souhaite avancer l'idée que le night-club et la discothèque représentent, au cours des années 1960 et au début des années 1970, bien plus qu'un programme architectural. Pour la néo-avant-garde de cette période, certains projets voués au divertissement musical assument un rôle central dans leur recherche stylistique et fonctionnelle, et constituent dans certains cas l'image d'une inspiration idéologique. La discothèque symbolise une manière de penser l'espace architectural et d'organiser la ville. Le choix certainement non exhaustif des travaux qui vont suivre trace une progression autant au niveau de l'échelle représentée, que dans l'implication idéologique des projets. Le Piped Environment introduit une réflexion sur le progrès de la technologie audio et son impact sur la perception des usagers. La recherche sur les Piper témoigne d'une curiosité pionnière de la part des architectes « radicaux» italiens pour des programmes novateurs dans l'enseignement de l'architecture comme le night-club. D’autres exemples de discothèques multimédias témoignent de l'attrait pour ces programmes de la part d'artistes intéressés par la construction d'environnements à sensations. Enfin, deux projets visionnaires de villes empruntent des images relatives aux discothèques pour repenser idéalement la structure de la société. L'ensemble de ces travaux illustre comment l'acte de bâtir, à partir de ces recherches, n'aboutit pas à un résultat formel figé, mais plutôt à une succession d'expériences et de stimulations perceptives. Au-delà de son aspect formel, l'architecture de ces projets expérimentaux est caractérisée par la construction d'atmosphères artificielles changeantes par l'intermédiaire de dispositifs technologiques et d'effets environnementaux. Cette transposition du rôle de l'architecture de l'objet bâti à l'expérience vécue transforme immanquablement le rôle de l'usager. D’objet réceptif plongé dans des environnements

The Story of the Song and Dance that Changed the World, Boston et Londres, Faber and Faber, 1995, p. 53 . 
multimédias, il devient, dans les dernières visions urbaines qui vont suivre, le principal aménageur des lieux qu’il habite.

\section{PIPED ENVIRONMENT}

It seems to have all started with Bell, Baird, Faraday, and the rest, although I doubt if they had thought of it in this way. What they did was to discover the facilities which have led to the Piped Environment. The immediacy of electrical response gave independence from the sun for light and heat and freed up many other situations in which the time lag of reaction had become an embarrassing restriction. Then the transmission of sound for communication made for an infinite expansion of the available information and exchange services ${ }^{3}$.

Pour Denis Crompton, un des membres du groupe d'architectes anglais Archigram ${ }^{4}$, le Piped Environment fit son apparition au temps de la génération de ses parents, à l'époque de l'invention des systèmes d'enregistrement et de diffusion sonores. À l'origine, la musique enregistrée était transmise par radio ou par ligne téléphonique. Elle créait un environnement individuel hautement personnalisé, qui ne s'imposait pas aux autres s'ils n’étaient pas branchés à ces dispositifs. Dès que les amplificateurs et les haut-parleurs furent inventés, cette qualité d'écoute individuelle déclina, nous plongeant dans un monde d'excès sonores collectifs ${ }^{5}$. L'utilisation des écouteurs, ou l'écoute individuelle, fit son retour, toujours selon Crompton, avec l'invention des radios transistor portables. «The astronaut cult so that the hi-fi man can now sit in oblivion extending himself in full frequency stereo sound without including the block in his experience $^{6}$.» En 1972, quand le texte The Piped Environement fut publié, le walkman n’avait pas encore été inventé, mais la technologie était prête à laisser Crompton imaginer une immersion dans des environnements sonores portatifs. En 1979, quand Sony produit le TPS L2, le premier modèle de walkman, le «culte de l'astronaute » devient une réalité à succès, et l'écoute individuelle et mobile, une

3. Dennis Crompton, «The Piped Environnement» dans Peter Cook, Warren Chalk, Dennis Crompton, David Greene, Ronald Herron et Michael Webb (dir.), Archigram, Londres, Studio Vista, 1972, p. 83.

4. Fondé en 1961, le groupe Archigram est composé de Warren Chalk, Peter Cook, Dennis Crompton, David Greene, Ron Herron et Michael Webb.

5. «This individual quality declined until we were all involved in the hi-fi nut's world of 100 watt Vorteixons and Tannoy drive units, whether or not this was the form of extension of our experience we were after », ibid., p. 83-84.

6. Ibid., p. 84 . 
pratique de tous les jours. Crompton, comme tous les membres d'Archigram, croyait dans le potentiel offert par la technologie pour repenser le rôle de l'architecture et de la ville. Plus qu'un objet physique, l'architecture désignait la construction d'environnements, et l'environnement, l'extension de l'expérience humaine. La technologie représentait donc cette possibilité d'extension de l'expérience humaine. "If the environmental business is concerned with the extension of man's experience then the means of achieving this is by pushing current technology ${ }^{7}$.»

Depuis l'avènement de la modernité, les architectes ont considéré le progrès technologique comme une possibilité de s'affranchir de la domination de la nature. Comme l'affirme Crompton, l'électricité a offert l'autonomie vis-à-vis du soleil pour l'alimentation en lumière et en chaleur, mais elle a aussi contribué, avec l'invention des systèmes d'enregistrement, à abolir les barrières spatiales et temporelles qui limitent la transmission du son. Grâce à la technologie électroacoustique, le son, comme la musique, n'est plus juste l'effet instantané d'un mouvement mécanique, mais aussi un phénomène reproductible à travers l'espace et le temps ${ }^{8}$. Plusieurs conséquences se dégagent de cette révolution technologique, mais ce qui frappe avant tout, c'est que la pratique de l'écoute musicale devient progressivement une constante de tous les jours. Haut-parleur, amplificateur, radio, stéréo: grâce à ces objets, la musique s’insinue dans la maison, au bureau, au supermarché ou dans l'ascenseur, sans plus besoin d'une performance en temps réel. De plus, l'écoute de différents genres de musique populaire devient, au cours du $20^{\mathrm{e}}$ siècle, une activité répandue qui n'appartient plus uniquement au cercle des habitués du concert. Des lieux comme le nightclub et la discothèque accueillent cette forme de divertissement très appréciée des amateurs du monde de la nuit.

\section{Ibid.}

8. «The ease which records travel in space and time has enabled the continual crossover and growing globalization that characterize the post-war popular music. While not quite a "music hall without walls," recording technology does trespass on the borders of neighborhood and nation. Significantly, it also traverses time: records are now readily available and longer-standing than live music; they efficiently distribute and preserve sound. It was in exploiting the time and space-binding characteristics of recording that disc cultures acquired distinction ", Sarah Thornton, Club Cultures: Music, Media and Subcultural Capital, Londres, Verso, 1995, p. 70. 


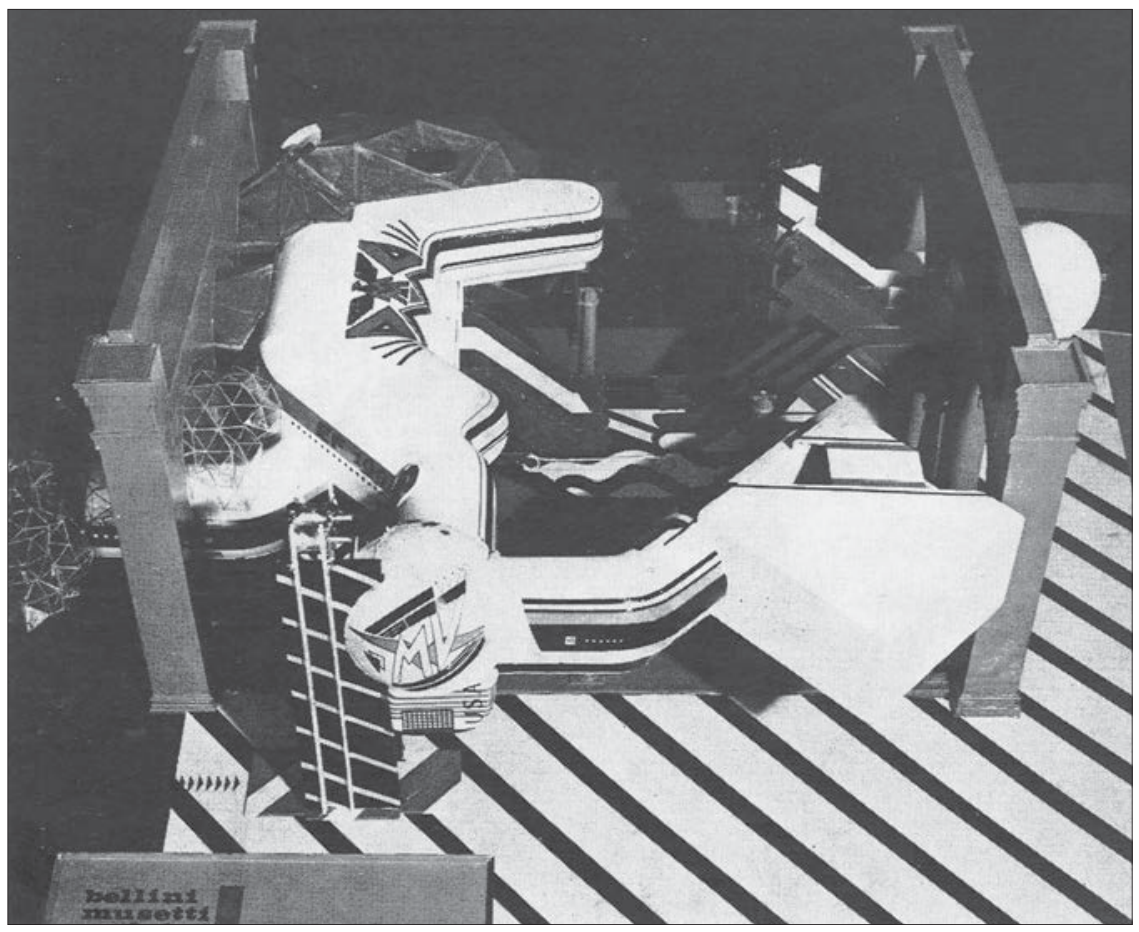

Fig. 1: Bellini, Musetti et Pedrini, projet d’étudiants réalisé dans le cadre du cours du professeur Leonardo Savioli «Spazio di Coinvolgimento» (1966-1967), dans Leonardo Savioli et Adolfo Natalini «Spazio di coinvolgimento », Casabella, n 326 , juillet 1968, p. 35 .

\section{PIPER}

À l'origine de la néo-avant-garde «radicale», au moment des dernières années de formation à la faculté d'architecture de Florence, les étudiants - futurs architectes « radicaux » ${ }^{9}$ - avaient suivi un cours particulièrement significatif pour les idées qui allaient se développer par la suite. Dans un contexte d'enseignement très académique, comme celui de l'Université de Florence, et en particulier dans la période qui précède 1968, le professeur Leonardo Savioli organisa un studio de projet d'aménagement d'intérieur sur le thème du «spazio di coinvolgimento »

9. Parmi les étudiants participant au cours, on peut nommer: Fabrizio Fiumi et Paolo Galli, fondateurs du groupe 9999, Dario Bartolini, futur Archizoom, Poli et Magris, futurs Superstudio, Carlo Bachi de UFO, Alberto Breschi et Giuliano Fiorenzuoli cofondateurs du groupe Zziggurat, et encore Lorenzo Barbieri, Massimo Cardini et Guido Coggiola, personnalités importantes dans les années qui suivent. Voir Paola Navone et Bruno Orlandoni, Architettura «radicale», Segrate, Documenti di Casabella, 1974, p. 25. 
- traduisible en «espace de participation» - pendant l'année 1966-1967 $7^{10}$. Les jeunes diplômés Adolfo Natalini et Paolo Deganello, respectivement futurs membres des groupes Superstudio et Archizoom ${ }^{11}$, assistèrent Savioli, et les architectes Pietro Derossi - futur membre du groupe Strum ${ }^{12}$ - et Maurizio Sacripanti participèrent au projet en tant que critiques extérieurs. Parmi les différents programmes dédiés à la distraction et au divertissement, un thème fut particulièrement exploré, celui des Piper. Piper vient du nom d'un local - Piper Clubqui ouvrit ses portes à Rome en 1965 et devint mythique pour les adeptes de la «dolce vita» locale ${ }^{13}$. Situé dans un ancien cinéma, le Piper Club s'inspirait, dans son style et sa programmation musicale, de la scène beat anglaise. Des œuvres pop d'Andy Wharol, Claudio Cintoli et Mario Schifano étaient exposées aux murs du local, et une lumière stroboscopique était projetée sur la piste de danse. Plusieurs stars italiennes et internationales vinrent s'exhiber dans ce berceau de la mondanité romaine. Cet exemple devint très vite un modèle, inspirant une production de projets particulièrement inventifs. La recherche de flexibilité spatiale était au cœur de l'intérêt de Savioli pour les Piper. Les étudiants étaient, à cette époque, très informés des développements de la culture pop anglaise, et notamment de la production de leurs confrères Archigram. Ils empruntèrent, d'une part, le principe d'utilisation de la technologie comme icône fonctionnelle, esthétique, et force motrice du projet, et d'autre part, une méthode de composition dégagée de la question de l'échelle. Dépaysement, translation de l'échelle, assemblage, montage, décomposition sont les méthodes qui étaient adoptées pour composer des modèles-objets qui se posaient eux-mêmes comme des «objets pop finis », avant même de renvoyer à une possible réalisation concrète ${ }^{14}$. On retrouve

10. Leonardo Savioli et Adolfo Natalini, «Spazio di coinvolgimento», Casabella, $\mathrm{n}^{\circ} 326$, juillet 1968, p. 32-45.

11. Les deux groupes se forment pendant l'hiver 1966-1967. Les membres de Superstudio étaient: Gian Piero Frassinelli, Alessandro Magris, Roberto Magris, Adolfo Natalini et Cristiano Toraldo di Francia. Le groupe Archizoom, quant à lui, se composait de: Andrea Branzi, Gilberto Corretti, Paolo Deganello, Massimo Morozzi, Dario Bartolini et Lucia Bartolini.

12. Pietro Derossi, Giorgio Ceretti et Riccardo Rosso formèrent le groupe Strum en 1971 .

13. Le Piper Club naît de la volonté des entrepreneurs Giancarlo Bornigia, Amerigo Crocetti et Pier Gaetano Tornelli. Installé au 9 Via Tagliamento, à Rome, ce grand nightclub connaît très rapidement un grand succès et une réputation internationale.

14. «L'adesione all'arte Pop del resto si manifesta, oltre che nella scelta del repertorio formale e nel conseguente suggerimento di precise scelte strutturali, anche nel tentativo di trasporne le metodologie di lavoro all'interno dei procedimenti compositivi 


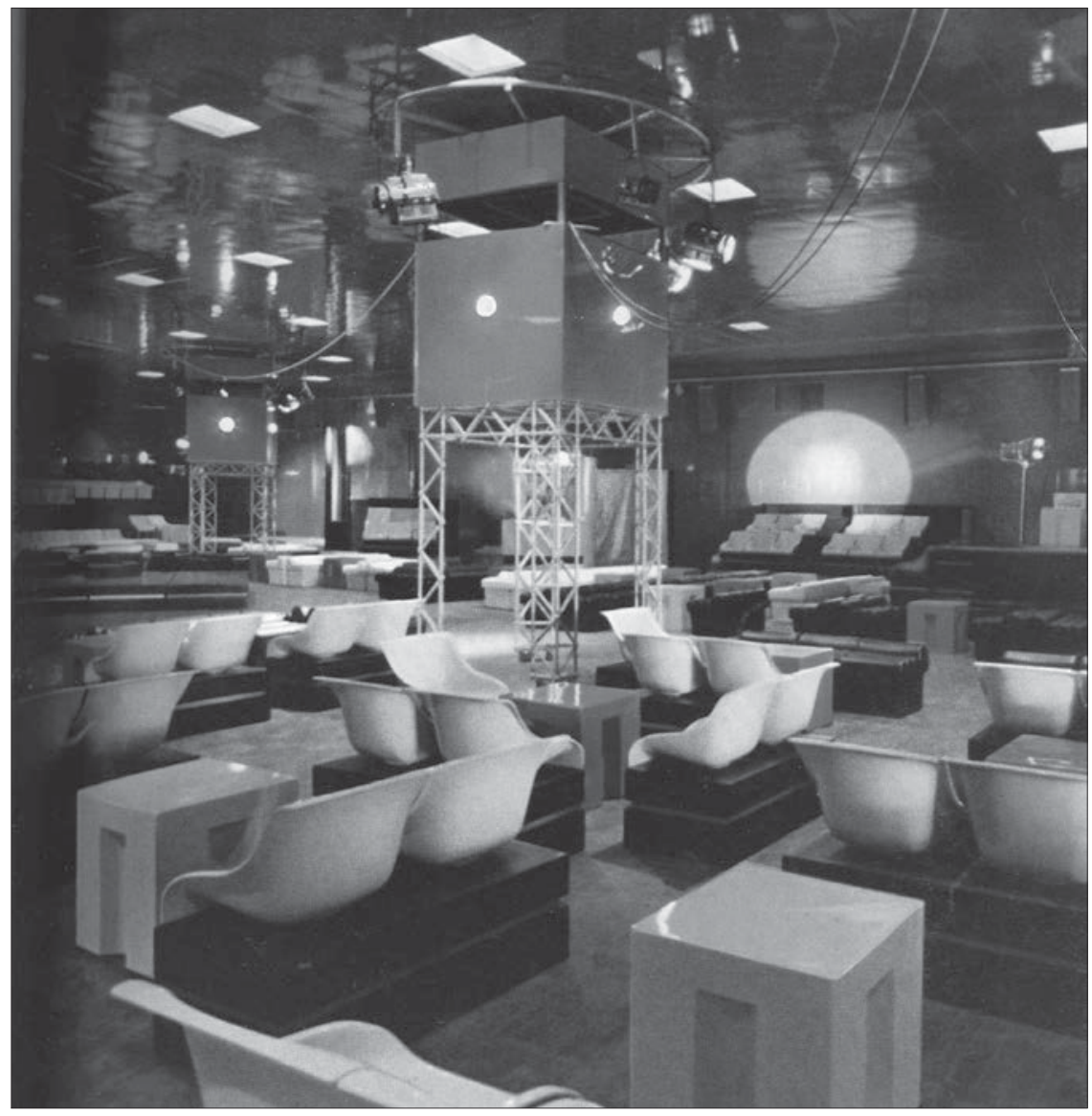

Fig. 2: Pietro Derossi et Giorgio Ceretti, L'altro mondo club, Rimini (Italie, 1967), dans «Divertimentifici », Domus, $n^{\circ} 45^{8}$, janvier 1968, p. 18.

dans leurs projets d'études un vocabulaire commun aux architectes pop anglais constitué de structures réticulaires, coques subtiles, membranes et volumes pneumatiques.

Très peu de temps après cette année de recherche à l'université, certains des participants au cours ont été amenés à répondre à de véritables commandes de night-clubs dans différentes villes d'Italie. En 1968, la revue Domus publie

specifici dell'architettura: spaesamento, traslazione di scala, assemblaggio, montaggio, scomposizione sono dei metodi che vengono adottati per comporre dei modelli-oggetto che si pongono essi stessi come oggetti pop finiti, prima ancora di rinviare ad una loro possibile realizzazione concreta ", Navone et Orlandoni, 1974, p. 26. 
un article intitulé «Divertimentifici ${ }^{15}$ » - traduisible en Divertissementeries sur deux nouveaux projets réalisés: le Piper-Pluriclub de Turin et l’Altro Mondo Club de Rimini, des architectes Pietro Derossi - qui assista Savioli lors du cours mentionné ci-dessus - et Giorgio Ceretti, lui aussi futur membre du groupe Strum. Les deux clubs étaient conçus sur le principe d'une boîte conteneur d'ambiances changeantes et modulables en fonction des nécessités, qui pouvaient aller de la clientèle à la programmation offerte. Dans un espace rectangulaire, le volume pouvait être plusieurs fois modifiable grâce à la présence d'équipements mobiles. Les chaises, les cimaises, les tours de soutien d'équipements techniques étaient toutes posées sur des roues et, au besoin, déplacées. Au lieu de proposer des espaces fixes, ces lieux étaient pensés comme des «machines sonores", comme on les avait dénommés. Du théâtre à l'exposition d'art, en passant par le concert et la «disco », ces espaces accueillaient différents types d'événements. Du beat au hippy, cette architecture, aussi définie comme «swinging» (sic), répondait rapidement aux styles et aux modes du moment.

En 1969, le groupe Superstudio réalise le Mach 2 dans une vieille cave auparavant inondée de Florence ${ }^{16}$. L'idée de boîte fermée de l'extérieur et conteneur d'effets atmosphériques - sons, lumières, vapeurs - est ici poursuivie. Carrément enterré, le volume intérieur était traversé par un réseau d'implantations visibles. Câbles, ampoules et haut-parleurs marquaient l'identité du projet en exaltant son infrastructure technique. Citant l'exemple d'un sousmarin devenu mythique dans l'imaginaire de l'époque - le Yellow Submarine des Beatles (1968) -, le projet était défini par le groupe comme un conte de lumières colorées qui se réfléchissaient sur les surfaces de la céramique noire posée au sol et sur les hublots en miroir ${ }^{17}$. Le milieu pop musical anglais devenait une des références pour ce mouvement d'architectes qui souhaitaient libérer Florence, ou bien ce berceau de la culture classique, du poids de l'histoire. D'ailleurs, la terrible inondation de 1966, qui dévasta beaucoup d'œuvres d'art de la ville, avait été interprétée par certains comme le signe représentatif d'un tournant radical qui poussait à chercher des repères plutôt vers d'autres horizons que vers les monuments du passé ${ }^{18}$.

15. Tommaso Trini, «Divertimentifici», Domus, n ${ }^{\circ} 458$, janvier 1968, p. 13-16.

16. Superstudio, «Tre architetture nascoste », Domus, n 473, avril 1969, p. 18-22.

17. Ibid.

18. Arata Isozaki avança l'idée d'une connexion entre les deux événements: «Alla fine del 66 ci fu l'alluvione di Firenze e la mostra della "Superarchitettura" con cui nacquero l'Archizoom e il Superstudio... forse gli storici dell'architettura radicale non hanno mai meditato abbastanza su tale straordinaria concomitanza di eventi, ma se ne 


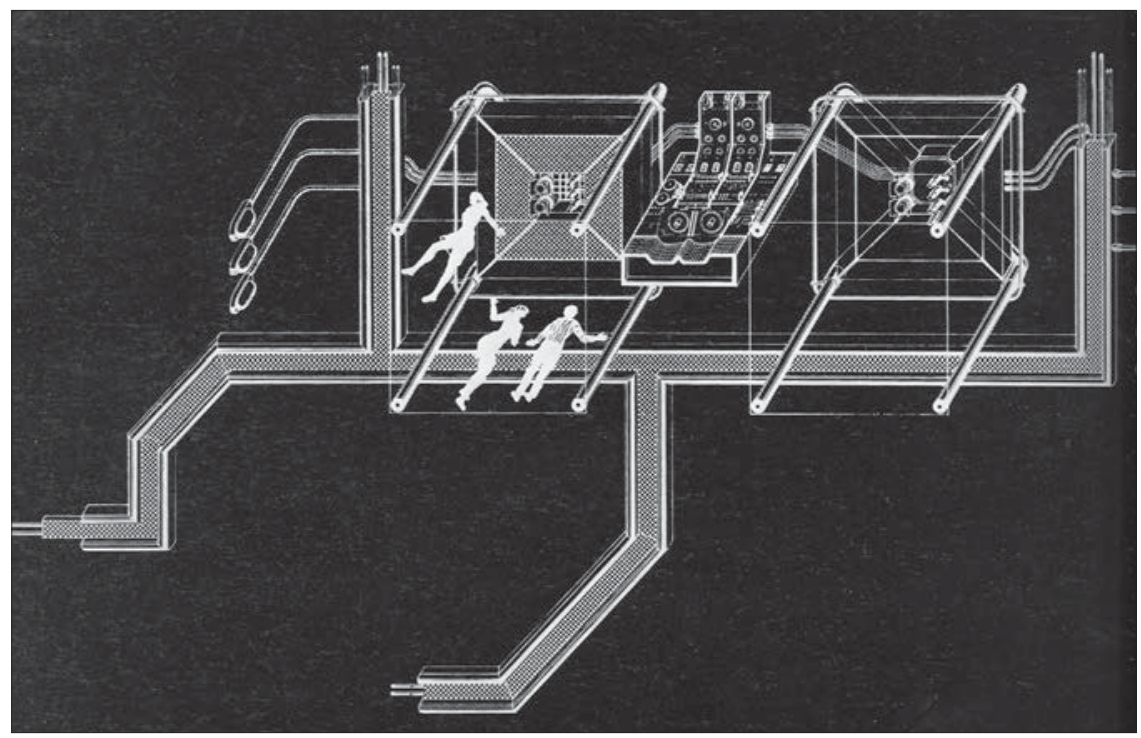

Fig. 3: Superstudio, Mach 2, Florence (Italie, 1968), dans «Superstudio: The architetture nascoste», Domus, $n^{\circ} 473$, avril 1969, p. 29.

\section{L'ART EN « BOÎTE »}

Si ces premiers projets impliquaient le plus souvent la présence d'une performance musicale, rentrant plutôt dans la catégorie des night-clubs, la discothèque proprement dite remplace définitivement la présence humaine des musiciens par une diffusion de musique enregistrée, tout au plus réglée et manipulée par une nouvelle figure légendaire, celle du disc-jockey orchestrant les boutons d'une platine. La discothèque, en français appelée aussi «boîte de nuit» ou «boîte», est un lieu de stimulations sensorielles par des systèmes de contrôle de l'environnement. Ces ambiances artificielles sont produites à l'intérieur d'une enveloppe close par des réseaux d'équipements techniques qui génèrent lumière, son, et contrôlent la circulation d'air.

Un autre projet, fruit de la recherche néo-avant-gardiste, cette fois-ci américaine, vient démontrer la puissance de l'image de la discothèque comme source d'inspiration. Le Pavillon Pepsi a été construit à Osaka lors de l'Exposition universelle de 1970, manifestation particulièrement significative en matière d'expérimentation technologique et d'application à l'architecture. Le vice-

accorse, con sottile intuito orientale, Arata Isozaki...», Adolfo Natalini, Alessandro Poli et Cristiano Toraldo di Francia, «Viaggio con la matita fra gli artefatti del mondo contadino », Modo, $\mathrm{n}^{\circ} 7,1978$. 


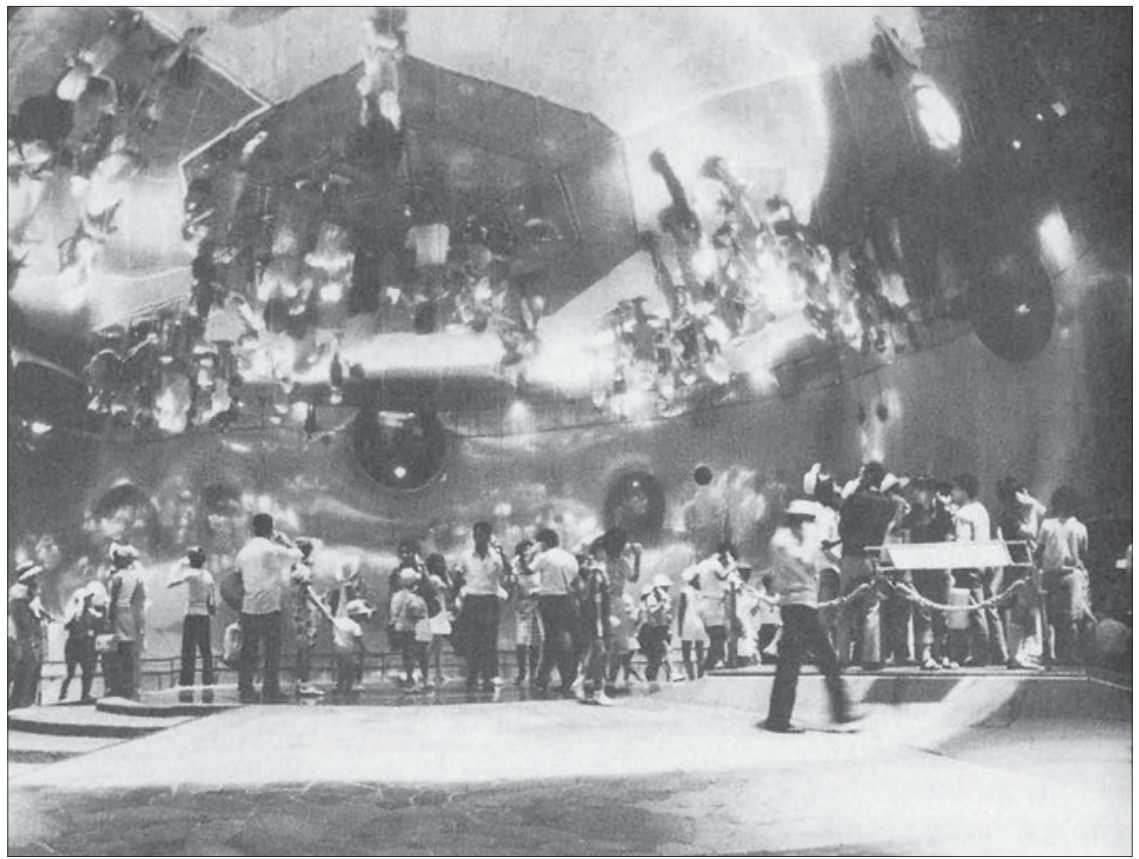

Fig. 4: Experiments in Art and Technology, Pavillon Pepsi (Osaka, 1970), dans Billy Klüver, Julie Martin et Barbara Rose, Pavillon, New York, E. P. Dutton, 1972, p. 248.

président marketing pour la firme Pepsi, David Thomas, invita Experiments in Art and Technology et le propriétaire d'une célèbre discothèque new-yorkaise de l'East Village - Electric Circus - à déposer leurs propositions respectives pour le pavillon qui, selon son souhait, devait incarner une sorte de «grande discothèque multimédia». Le projet retenu a finalement été celui d'E. A. T. Fondé en 1966, par les ingénieurs Billy Klüver - ancien ingénieur chez Bell Telephone Laboratories - et Fred Waldhauer, et les artistes Robert Rauschenberg et Robert Whitman, E. A. T. combinait l'art et la science pour la création d'espaces et d'installations ouvertes au public.

Le Pavillon Pepsi était constitué d'un dôme de $120 \mathrm{~m}$ de diamètre à l'extérieur et $90 \mathrm{~m}$ à l'intérieur, conçu par l'architecte John Pearce et construit par la firme d'architectes-ingénieurs Takanata Komunten. Un groupe d'artistes ont collaboré à sa conception et à sa réalisation suivant le concept promu par Robert Rauschenberg: la création d'une expérience multisensorielle par un «environnement invisible». La liste des personnes ayant participé à la réalisation du projet est interminable et comprend plus d'une quarantaine d'ingénieurs ainsi qu'une vingtaine d'artistes. Une synergie exceptionnelle de compétences 
au service d'une expérience totalisante pour le spectateur. Différents éléments composaient cet environnement ${ }^{19}$. La Fog Sculpture de la sculptrice Fujiko Nakaya était un brouillard provoqué par des vapeurs d'eau qui enveloppaient le pavillon; le Suntrack de Forrest Myers était une image triangulaire projetée sur le toit du pavillon qui suivait le trajet du soleil; les sept Floats de Robert Breer étaient des sculptures flottantes qui se déplaçaient sur la Plaza, à travers le brouillard, grâce à un système de roues pivotantes cachées, en émettant des sons. Le Light Frame de Forrest Myers était composé de quatre perches verticales délimitant les quatre coins de la Plaza, au bout desquelles des faisceaux lumineux de xénon créaient des jeux de lumière. Le tunnel incliné de l'entrée, pour sa part, était constitué de treize sections fabriquées avec des matériaux différents - de l'asphalte à la pelouse - et à l'intérieur, la Clam Room - en forme de coquille de palourde - était composée d'un plancher mou et diffusait des rayons laser de lumières multicolores sur le spectateur. Au centre, le Miroir, une sphère dont les parois étaient composées d'une fine pellicule de Melinex qui était soutenue par un système d'air à propulsion. L'image du spectateur et du lieu était projetée sur ces parois, créant une sensation de flottement. Enfin, un dispositif d'écoute était fourni à chaque spectateur : il procurait une variété de sons saisissants selon l'endroit de la déambulation et en fonction des différents matériaux posés au sol. L'ensemble des effets sonores avait été créé par David Tudor, en collaboration avec Robert Whitman. Un système constitué de trente-sept haut-parleurs, alimenté par trente-deux différentes entrées et géré par un panneau de contrôle général, créait techniquement plusieurs expériences sonores à partir de trois principes perceptifs : 1 . La ligne sonore : un signal qui bougeait d'un haut-parleur à un autre à une vitesse de $1 / 3$ de seconde. 2 . Le point sonore: un seul signal émis par un haut-parleur placé à un point spécifique du dôme. 3. L'immersion: ou le son environnemental, des signaux provenant de partout ${ }^{20}$. Dans un bain de différentes sensations, le voyage du spectateur à l'intérieur du pavillon restait marqué par une série d'expériences changeantes et éphémères. Pour Billy Klüver, le pavillon était une œuvre d'art par son unité et son intégrité. «The Pavilion was a work of art with its unity and integrity, as well as a new unexplored theatre

19. Une histoire du projet et la description exacte des dispositifs du pavillon sont données par Sylvie Lacerte, www.olats.org/pionniers/pp/eat/eat.php (dernière consultation le 27 avril 2010).

20. D'autres descriptions techniques et détaillées de l'ensemble des dispositifs sont données par Elsa Garmire, «An Overview», dans Billy Klüver, Julie Martin et Barbara Rose (dir.), Pavilion: Experiments in Art and Technology, New York, E. P. Dutton \& Co., Inc., 1972, p. 173-206. 
and concert space, a recording studio for multichannel compositions and a field laboratory for scientific experiments ${ }^{21}$.»

Du point de vue de l'architecte, John Pearce, les artistes s'étaient déclarés dès le début «anti-Expo », et affirmaient que le meilleur pavillon serait un nonpavillon : «An ideal solution would have been a pavilion that could disappear ${ }^{22}$.» Plusieurs propositions physiques, raconte l'architecte, avaient alors été faites pour répondre à cette idée de disparition, comme des entrées trous, des fontaines d'eau et des jeux de lumière qui auraient caché le pavillon dans sa forme et sa structure. Le rôle de l'architecte était alors celui de gérer la circulation du public à l'intérieur de cet espace et d'en assurer le fonctionnement: «Service areas, public toilets, offices, etc. were never a problem, so, consequently, they were never discussed. They were expected to disappear and they did ${ }^{23}$. » À l'intérieur, relate encore Pearce, la structure de support, l'air conditionné, le circuit électrique, etc. devaient être intégrés au projet, l'architecte avait une liberté totale, avec une seule contrainte, celle de ne pas modifier les intentions des artistes. Entre l'ingénierie et l'art, l'architecte devenait ainsi une sorte d'interface de communication et son œuvre, un objet absolument invisible. Le Pavillon Pepsi, tout entier, est alors un lieu d'expérience pour le spectateur. La lumière, le son et la sensation tactile définissent cet environnement spécifique; ils remplacent enfin l'architecture bâtie.

La discothèque a été le lieu de diverses expériences sensorielles menées par d'autres artistes comme Jean-Paul Mousseau, qui, entre 1966 et 1976, réalise quatre discothèques dans la ville de Montréal: des «boîtes à ambiance totale » réalisées par le recours au son, à la lumière, et avec l'idée d’y ajouter des parfums $^{24}$. Ou encore le Voom Voom, night-club réalisé à Saint-Tropez en 1966 par Nicolas Schöffer, en collaboration avec l'architecte Paul Bertrand. Schöffer utilise des dispositifs déjà existants, comme le prisme et le microtemps, pour créer des effets luminodynamiques qui agissaient comme des «massages neuronaux» sur le public. Voom Voom n'était pour Schöffer qu'une application à petite échelle de son idéal urbain. En 1969, il publie un projet visionnaire de «ville cybernétique », une extension du principe de contrôle des environnements

21. Ibid., p. xiv.

22. John Pearce, «An Architect's View», dans Klüver, 1972, p. 256.

23. Ibid., p. 258.

24. Pour en savoir plus sur ces réalisations, voir André G. Bourassa, «Jean-Paul Mousseau: pour un nouvel espace scénique», Études françaises, vol. 34, n²-3, 1998, p. $125-139$. 
et de conditionnement des usagers par l'utilisation diffuse de la cybernétique ${ }^{25}$. Le projet de François Dallegret, en collaboration avec l'architecte Joseph Baker, pensé en 1968 pour un fragment de ville souterraine à Montréal, est pour sa part une proposition qui se situe entre l'idée de boîte à distraction et un idéal urbain plus étendu ${ }^{26}$. Palais Métro était un grand centre commercial auquel on pouvait accéder directement par le métro. Une structure tubulaire d'échafaudages offrait une grande flexibilité d'usage. Entre les magasins et les cafés, Palais Métro allouait des plateformes pour différents événements et spectacles: expositions d'art, concerts de jazz, groupes de musique rock'n'roll, spectacles de danse, musiciens de la rue, défilés de mode et tournages de films. Défini comme un «centre commercial d'avant-garde» et un lieu «anti-tradition», le projet de Dallegret, qui ne sera jamais réalisé, profite de sa localisation souterraine et renfermée pour assimiler la ville à une méga boîte de nuit combinant «the geometric forms and psychedelic colors of contemporary art ${ }^{27}$ ».

25. Nicolas Schöffer, La ville cybernétique, Paris, Éditions Tchou, 1969. Schöffer publiera quelques années plus tard un deuxième ouvrage sur sa théorie urbaine, sous un titre qui fait clairement référence à l'œuvre de Le Corbusier : Nicolas Schöffer, La nouvelle charte de la ville, Paris, Éditions Denoël-Gonthier, 1974. Dans un entretien au sujet de Voom Voom Schöffer répond:

«M.D. : Ne pensez-vous pas que ce complexe-loisir soit une forme de conditionnement du public?

S.N.: Si. Mais c'est un conditionnement esthétique. Tous les night-clubs sont des formes de conditionnement mais généralement par le bas. On ne peut pas s'opposer à cette forme de loisir qui, par opposition au loisir intellectuel, est plus propre à la détente et que j'appellerai «loisirs sexuels », mais on peut orienter le conditionnement, y introduire un élément esthétique. C'est ce que j'ai essayé de faire.

M.D. : Pourquoi avez vous réalisé cet ensemble?

S.N.: Par souci d'urbanisme. Il y a trois complexes d'urbanisme: travail, repos, loisir. J'ai pu réaliser ici un complexe-loisir, tel que je le souhaitais, sans aucune contrainte. Denise Miège et Nicolas Schöffer, «Les merveilleux mirages de Schöffer », Arts, 17 août 1966, s.p.

26. Joseph Baker, «Psychedelic Marketing: Palais Metro», Art in America, vol. 56, $\mathrm{n}^{\circ}$ 4, juillet-août 1968, p. 92-93. Le projet Palais Métro et les discothèques de Jean-Paul Mousseau ont été montrés à l'exposition Environnement total: Montréal 1965-1975, réalisée dans le cadre d'un séminaire de recherche de l'Université de Montréal (et organisée par le professeur Alessandra Ponte) qui a été présentée au Centre Canadien d'Architecture du 19 mars au 23 août 2009 .

27. Ibid. 


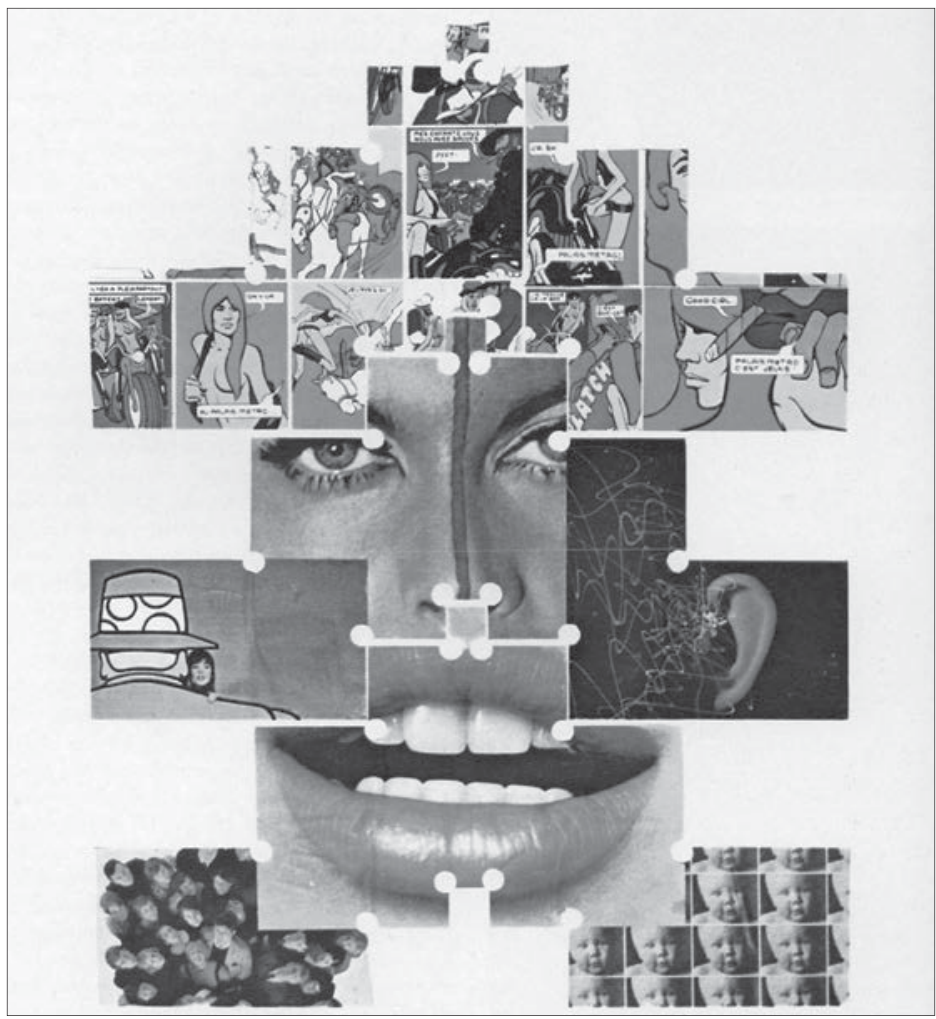

Fig. 5: François Dallegret, publicité pour Palais Metro (1967), dans Art in America, nº 4, juillet-août 1968, p. 92.

\section{JUKE-BOX URBAINS}

$\mathrm{Si}$, d'une part, la discothèque constitua un laboratoire pratique pour différents artistes, d'autre part, l'idéal du contrôle total des environnements habita aussi certaines positions théoriques dans le discours architectural. L'ouvrage de Reyner Banham, The Architecture of the Well-Tempered Environment, est à ce propos une référence fondamentale pour comprendre la quête d'une conjonction progressive entre la notion d'architecture et celle d'environnement contrôlé, notamment en ce qui concerne le climat ${ }^{28}$. Banham propose une lecture de la modernité du point de vue de la technique et s'éloigne de toute interprétation stylistique du fonctionnalisme en architecture. Pour le critique britannique, certaines figures du panorama américain, comme Frank Lloyd Wright et Louis Kahn, utilisèrent

28. Reyner Banham, The Architecture of the Well-Tempered Environment [1969], Chicago, The University of Chicago Press, 1984. 


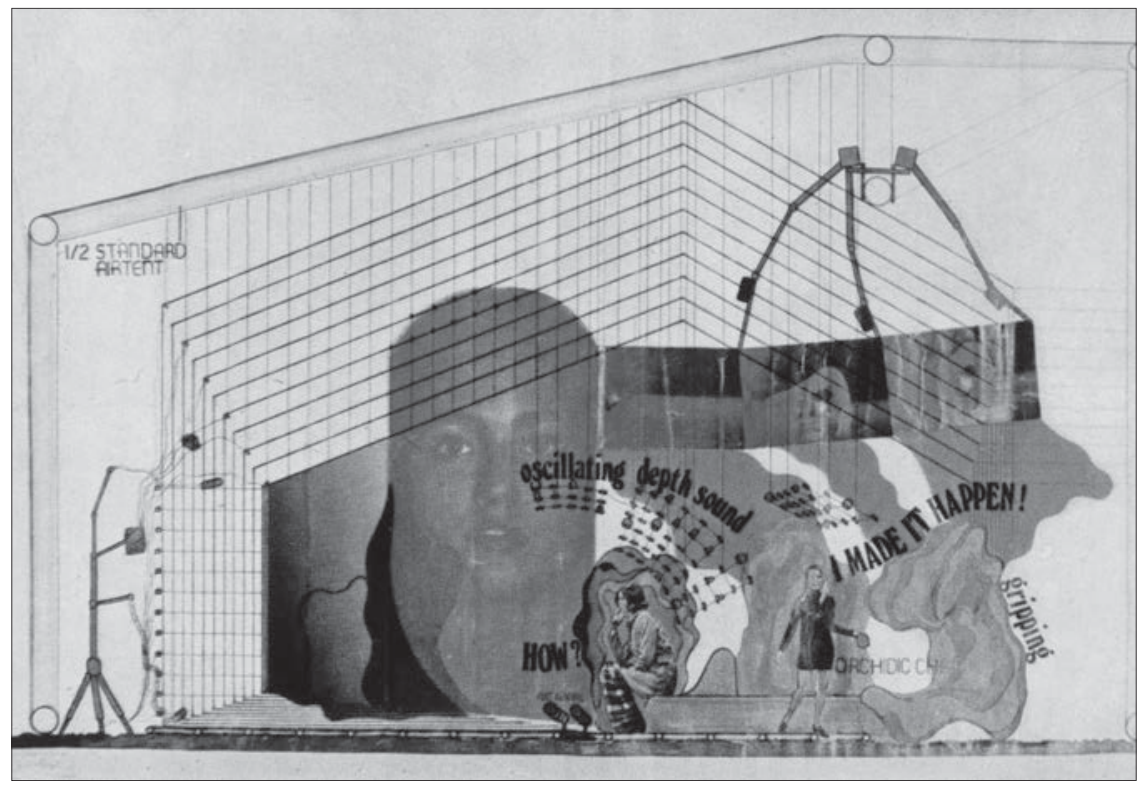

Fig. 6: Archigram, Audio-Visual Juke Box in Instant City, 1968.

la technique comme génératrice du projet, dans la forme comme dans l'usage, désignant la vraie face de l'architecture moderne. Lopposition, puis le passage, de la masse aux fluides, d'une architecture de la structure à une architecture de l'énergie, est l'aspect qui caractérise cette approche de l'architecture moderne dont il est, pendant les années 196o, un fervent porte-parole. La production de ses compatriotes d'Archigram était donc particulièrement chère à Banham ${ }^{29}$. Leur recherche, qui n'atteindra jamais l'état de la réalisation, représentait une version particulièrement «festive» de son attachement à la technologie.

Pour revenir plus spécifiquement au centre du sujet, le night-club, ou la discothèque, est parfois représenté dans les projets d'Archigram, mais ce qui devient plus significatif dans le travail du groupe, c'est que la ville en ellemême se révèle par certains aspects une sorte de «boîte» à grande échelle. Le projet d'Instant City (1968) est sans doute l'exemple le plus éloquent. Constitué d'un système d'équipement multimédia transportable par un dirigeable et

29. Banham publie plusieurs projets d'Archigram dans son ouvrage sur la mégastructure: Reyner Banham, Megastructure: Urban Futures of the Recent Past, Londres, Thames and Hudson, 1976. Le travail des étudiants de Savioli, le projet de François Dallegret et New Babylon sont aussi mentionnés dans son ouvrage. 


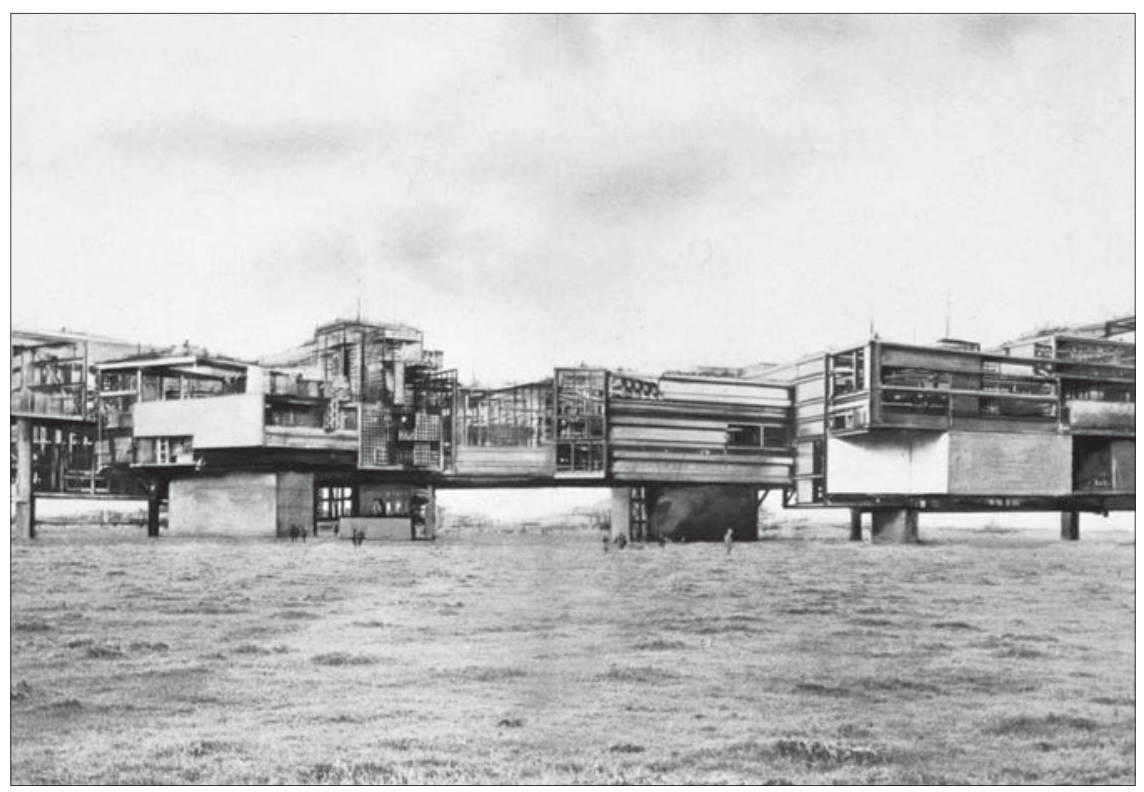

Fig. 7: Constant Nieuwenhuis, New Babylon, 1956-1972.

des camions, Instant City vient se greffer sur un centre urbain préexistant pour faire goûter aux habitants les bénéfices d'une métropole dynamique équipée d'un dense réseau d'information entre différentes communautés, mais aussi en lien direct avec la localité d'accueil. Des structures pneumatiques et des tentes supportent un système audiovisuel et des équipements d'éclairage électrique. Les Audio-Visual Juke Box sont des machines qui permettent aux habitants de se brancher sur un environnement choisi parmi une gamme de programmes audiovisuels. Il s'agit de robots capables de conditionner l'environnement selon la volonté des usagers. Ces machines descendent d'autres dispositifs prototypes, réalisés à Oslo en 1969, et appelés Soft Scene Monitor. À l'intérieur d'une cabine fermée, le public était assis dans un fauteuil confortable et immergé de tous les côtés dans un environnement audiovisuel sélectionné par ses soins. Par une sorte de télécommande insérée dans l'accoudoir du fauteuil, il choisissait ses préférences. L'image du juke-box renvoie à l'idée du divertissement musical, mais aussi au principe d'une multiplicité de choix offerts à l'usager. Le contrôle des environnements n'est pas imposé par un régisseur, ou bien par un disc-jokey - ou dans ce cas par l'architecte -, mais il vient du bas, du souhait de l'habitant. Instant City, avec ses dispositifs, représente une sorte d'interface technologique qui offre la possibilité de communiquer, de s'informer et de se divertir librement. 
Le même souci social habite, de manière encore plus décisive, le projet de New Babylon sur lequel l'architecte situationniste Constant Nieuwenhuis travaille de 1956 à 1974. Ce projet de ville part d'une position tout d'abord politique. L'Homo ludens - nom de baptême de l'habitant de New Babylon - ne doit plus être contraint par une définition statique des espaces qu'il occupe. À la différence de l'homme de la société actuelle, essentiellement utilitariste, le New-Babylonien habite dans une société ludique et mobile où toute relation sociale doit être libre et variable. Cette souplesse idéologique se reflète en architecture par un système d'urbanisme unitaire, fruit d'une créativité changeante et collective. Une grille continue, ou macrostructure, occupe de façon régulière la totalité de la surface terrestre, à la façon d'un échafaudage horizontal, et est indépendante de toute intention esthétique. À l'intérieur, on retrouve des secteurs - des micro-ambiances - déterminés par les usagers qui peuvent choisir leur environnement de manière créative et variée. Le principe de ces zones fermées est comparable à celui des «boîtes » : isolées de l'atmosphère extérieure et gérées par un système de contrôle technologique, elles permettent une maîtrise totale et arbitraire de l'ambiance intérieure, en totale indépendance des conditions naturelles existantes. Persuadé que les effets produits par un environnement sont capables de conditionner l'état psychique de manière plus incisive que l'aménagement spatial des lieux, Constant offre aux habitants de New Babylon une complète liberté de décision. À nouveau, la technologie joue un rôle déterminant; sans elle, la réalisation de ce collectivisme expérimental n’aurait en effet pas lieu. L'architecte est remplacé par une volonté plurielle qui se sert des capacités révolutionnaires des systèmes de contrôle des ambiances. Dans le discours de Constant, on retrouve étonnamment l'image du juke-box, ce «meuble musical» qui, une fois actionné, conditionne, stimule et divertit le New-Babylonien, auteur de son propre environnement. Comme un jukebox pluri-sensoriel, les ambiances de cette ville déterminent la qualité de cette nouvelle architecture multipliée à échelle urbaine, voire globale.

À New Babylon, la climatisation ne sert pas uniquement à recréer, comme dans la société utilitariste, un climat «idéal», mais à varier, dans la mesure du possible, l'ambiance. Quant à la télécommunication, elle ne sert pas seulement, ou principalement, des intérêts d'ordre pratique. Elle est au service d'activités ludiques, elle est une forme de jeu. Pour mieux comprendre, prenons l'exemple d'un café de quartier, un café bien tranquille dont l'atmosphère s'animerait soudain quand quelque nouveau venu aurait jeté une pièce de monnaie dans le juke-box. À New 
Babylon, chacun peut, à chaque instant, où qu'il se trouve, changer l'ambiance en réglant différemment le volume sonore, l'intensité lumineuse, l'ambiance olfactive, la température ${ }^{30}$.

\section{L'UTOPIE DE LA DISCOTHÈQUE}

En observant cette succession de projets, on retrouve des thèmes récurrents. Larchitecture, dans la plupart des projets mentionnés, assume son importance principalement par son fonctionnement interne et non pas par son image extérieure. La concentration d'infrastructures à l'intérieur des lieux de distraction devient, à l'échelle urbaine, une macro ou mégastructure soutenant des réseaux technologiques diffus. L'environnement à l'intérieur de ces architectures se construit perpétuellement, et de manière variable, avec la participation plus ou moins directe de l'usager. Le langage de représentation utilisé emprunte le plus souvent à l'imaginaire pop et évoque par ses modes de fabrication la dimension du jeu et du divertissement. Ces diverses expériences partagent donc des objectifs, des méthodes et des expectatives communs. Mais pourquoi le night-club et la discothèque comme objet de recherche occupent-ils une place particulièrement cruciale au sein de la néo-avant-garde architecturale? Tout d'abord la mode des night-clubs et des discothèques marque la génération des architectes concernés en dehors de leur profession, et en tant qu'utilisateurs directs. L'univers musical pop, puis disco, né au cœur de certains de ces lieux légendaires, fabrique un imaginaire dans lequel ces jeunes architectes et artistes puisent leurs références et repèrent leurs héros. Ensuite, la place de la technologie joue sans doute un rôle crucial. La «boîte » est proprement un lieu de concentration de réseaux et d'infrastructures techniques. Pour la néo-avant-garde des années 1960, le progrès technologique constituait un vaste répertoire pour de nouvelles directions de recherche. La «boîte» permettait d'expérimenter la création d'environnements artificiels à une échelle concentrée. Le fonctionnement des flux intérieurs était, dans ce type de programme, primordial par rapport à l'aspect formel et au langage stylistique du projet. Enfin, cette génération qui succède à la grande vague internationale du Mouvement moderne cherche dans l'activité du divertissement une manière de s'évader des préceptes rigides, parfois dogmatiques, de ses pères. Dans une société qui traversait une période de bien-être exceptionnel, comme celle des trente glorieuses, l'industrie du plaisir occupait sans complexe

30. Constant Nieuwenhuis, «Technologie», dans New Babylon, Constant: art et utopie, textes situationnistes, édition établie et présentée par Jean-Clarence Lambert, Paris, Les Éditions Cercle d'Art, 1997, p. 96 (nous soulignons). 
ou pudeur la créativité des nouvelles générations d'architectes. La «société du spectacle», critiquée par Guy Debord en 1967 en tant que stade achevé du système capitaliste, était plutôt, dans cet ensemble de recherches, le contexte pour l'affirmation de nouvelles pratiques sociales émancipatrices. Sans renoncer à leur engagement politique, ces architectes et artistes des sixties trouvèrent des nouveaux cadres - plus proches de leur quotidien - à explorer, et poussèrent leur imagination parfois jusqu'à l'utopie d'une société marchant au rythme d'un tube de disco. 
